\section{Engineering in Translational Medicine}

W. Cai, Ed.

New York, NY: Springer, 2014, 999 pages, $\$ 329.00$

Translational medicine is a rapidly growing discipline in biomedical research and aims to expedite the discovery of new diagnostic tools and treatments by using a multidisciplinary, highly collaborative, bench-to-bedside approach. The recent progress in translational medicine has received tremendous attention over the last decade. However, until now, a comprehensive reference book that focuses on engineering and translational medicine had not been published anywhere in the world. The purpose of this book is to fill that gap and meet the urgent need. The editor is Weibo Cai, an associate professor in the Department of Radiology, Medical Physics, and Biomedical Engineering at the University of Wisconsin. This hardcover 999-page book, with high-quality images printed on highquality paper, is authored by about 80 scientists, many of whom are international leading experts in the field of translational medicine.

A broad spectrum of state-of-the-art engineering research in translational medicine is covered in the 44 chapters, which are well organized into 5 parts: "Cell and Tissue Engineering," with 6 chapters, "Genetic and Protein Engineering," with 10 chapters, "Nanoengineering," with 10 chapters, "Biomedical Instrumentation," with 4 chapters, and "Theranostics and Other Novel Approach," with 4 chapters.

The first parts deals with the use of cell and tissue engineering for translational medicine, including engineering and noninvasive imaging of stem cells and associated clinical applications, engineering of T-cells for cancer treatment, biomaterials for the delivery of therapeutic cells, tissue engineering of nerve grafts, tissue engineering of blood vessels, and gene-activated matrices for the repair of articular cartilage defects.

The second part is devoted to a broad array of areas related to genetic and protein engineering, including luciferases for assays and imaging, split reporter systems for molecular imaging of protein-protein interactions, engineering requirements for bioluminescence resonance energy transfer, antibodies, Affibody- and protein-based imaging and therapeutics, aptamers for biosensing and targeted delivery of imaging and therapeutic agents, and DNA vaccines for cancer therapy.
The third part focuses on the emerging topic of nanoengineering, covering nanoparticle-based delivery systems, biosensing, optical imaging, MR imaging, nuclear imaging of nucleic acid, and cancer therapy.

The fourth part relates to biomedical instrumentation, which has recently witnessed tremendous advances in engineering. Chapters 28-30 discuss the development of new imaging systems for various contrast agents, including next-generation PET detectors, photoacoustic imaging, and miniature imaging, and the last chapter is on preclinical radiotherapy systems for cancer treatment.

The last part of this book highlights theranostic and other novel approaches to translational medicine, including a comprehensive review of cancer theranostics with plasmonic nanobubbles, cellbased microfluidic assays, engineering of photomanipulatable hydrogel, and a new class of peptidomimetics.

Major strengths of this book include detailed procedures, workflow diagrams with cartoon illustrations, and detailed discussions of the connection between basic biologic science and engineering. It is this complementary relationship between new basic-biology discoveries and the precise, quantitative, and predictable nature of engineering that underlies the significant advances described in each chapter.

In conclusion, this book will serve as a guide through which scientists, students, clinicians, and bioengineers working in the areas of engineering or translational medicine can rapidly acquire the basic and current knowledge essential to clinical translation. We highly recommend this book to trainees, biomedical researchers, and clinical physicians. Researchers who want to translate their discoveries to the clinic will also find the book quite useful.

Min Zhou
Mei Tian*
*The Second Affiliated Hospital of Zhejiang
University School of Medicine
88 Jiefang Rd.
Hangzhou, Zhejiang 310009, China
E-mail: meitian@zju.edu.cn

Published online Jun. 11, 2015.

DOI: $10.2967 /$ jnumed.115.161984 